\title{
Pleurectomy through the triangle of auscultation
}

\author{
OJ LAU, S SHAWKAT \\ From the Thoracic Surgical Unit, Preston Hall Hospital, Aylesford, Kent
}

The aetiology of primary spontaneous pneumothorax is unknown, though several theories have been proposed. The formation and rupture of "blebs" in the lung are frequently associated with primary pneumothorax,${ }^{1-3}$ but the management of the condition remains controversial and depends on its severity and the patient's previous medical history. For definitive treatment pleurectomy still remains the treatment of choice. ${ }^{45}$ We have treated 25 young patients with primary spontaneous pneumothorax with apical pleurectomy through the auscultation triangle, without incision of the muscles of the chest wall. We have found that this approach has several advantages over a full thoracotomy.

\section{Operative technique and results}

Twenty-five young patients with primary spontaneous pneumothorax have been treated, of whom 15 were men. Ages ranged from 16 to 38 years. Six patients had had a persistent air leak for more than one week despite intercostal tube drainage, and the remainder had a past history of two or more episodes of recurrent spontaneous pneumothorax.

Patients are prepared and positioned as for a routine full posterolateral thoracotomy, with the scapula drawn forward. In this position the auscultation triangle is clearly defined, being bounded by the trapezius above, the latissimus dorsi below, and medial border of the scapula laterally. An $8-10 \mathrm{~cm}$ incision is made along the medial border of the scapula over the triangle and the muscle boundaries are freed by division of the fascia. The fascial sheath, which forms the floor of the triangle, is then incised to expose the sixth and seventh ribs and the intercostal space between them. A small thoracotomy is made through the bed of the seventh rib and enlarged gradually by further mobilisation of the surrounding muscles and fascia. The apical parietal pleura is stripped from the endothoracic fascia, the lung inspected, and any bulla or air leak dealt with accordingly. If a problem should arise requiring a larger exposure, the operation could be transformed into a full thoracotomy, but we have found this to be unnecessary. The pleural cavity is drained by one intercostal tube attached to an underwater-seal drainage bottle and the chest wall closed in three layers-periosteal, subcutaneous, and subcuticular.

There were no postoperative complications. The mean inpatient period after surgery was $4 \cdot 6$ days (range 4-6 days).

Address for reprint requests: Mr OJ Lau, 135 Coleraine Road, London SE3.
Outpatient follow-up for one to three years has shown no recurrence of pneumothorax.

\section{Discussion}

For most cases of primary spontaneous pneumothorax, observation, bed rest, or intercostal tube drainage are adequate; but for patients with persistent air leak, and for those with a history of recurrent attacks, some form of definitive treatment is necessary. Various methods have been recommended, from artificial obliteration of the pleural space with various chemicals or oils to the stripping of the parietal pleura and closure of the air leak through a formal thoracotomy.

In our experience, these forms of treatment are often associated with unnecessary pain and discomfort for the patient and may require a lengthy stay in hospital; moreover, some of the methods are followed by complications, which are well documented. ${ }^{67}$ We have found that our approach to pleurectomy has several advantages, especially

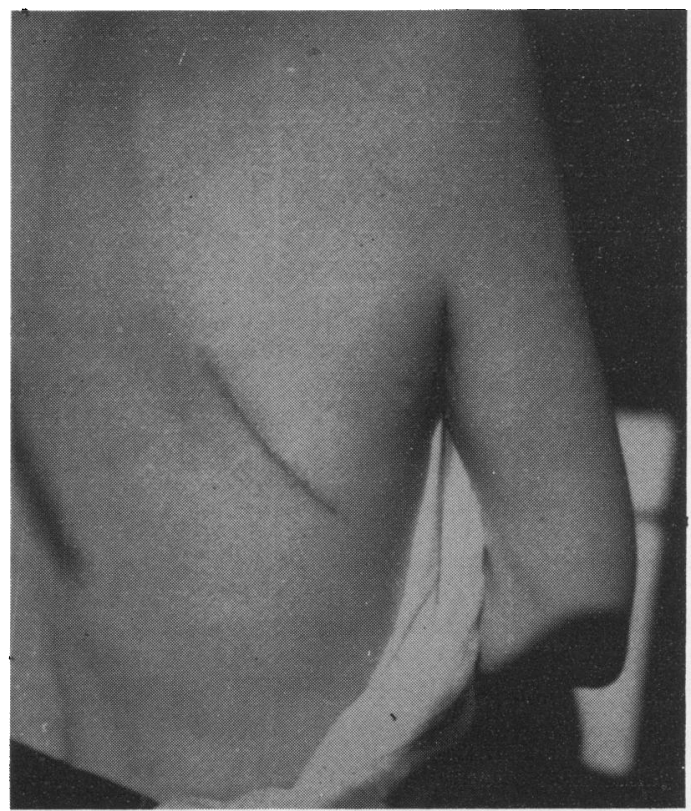

A cosmetically acceptable thoracotomy scar (30 days after operation). 
in younger patients, presumably because they have more resilient ligaments and mobile costovertebral joints, which allow adequate opening of the intercostal space. As no muscles are incised during the operation, the patient is usually fully mobile on the first postoperative day. This approach also shortens the hospital stay from a mean of $8 \cdot 5$ days (range 7-10 days) for a routine thoracotomy to $4 \cdot 6$ days. Cosmetically, it gives a more acceptable scar than does a full thoracotomy (fig).

\section{References}

' Lichter I, Gwynne JF. Spontaneous pneumothorax in young subjects. A clinical and pathological study. Thorax 1971;26:409-17.

${ }^{2}$ Singh SV. Current status of parietal pleurectomy in recurrent pneumothorax. Scand $J$ Thorac Cardiovasc Surg 1979;13:93-6.

3 Withers JNW, Fishback ME, Kiehl PV, Hannon JL. Spontaneous pneumothorax. Suggested etiology ancम्ठ comparison of treatment methods. Am J Surg 1964, 108:772-6.

4 Deslauriers J, Beaulieu M, Després J-P, Lemieux $M, \overrightarrow{0}$ Leblanc J, Desmeules M. Transaxillary pleurectomy, for treatment of spontaneous pneumothorax. $A n n-$ Thorac Surg 1980;30:569-74.

5 Ruckley CV, McCormack RJM. The treatment of spontaneous pneumothorax. Thorax 1966;21:139-44.

- Gobbel WG, Rhea WG, Nelson IA, Daniel RA. Spontaneous pneumothorax. I Thorac Cardiovasc $\mathrm{N}$ Surg 1963;46:331-45.

7 Jackson JW, Bennett MH. Chest wall tumour following iodized talc pleurodesis. Thorax 1969;28:788-93. 